\title{
Calibration of Parallel Bond Parameters in Bonded Particle Models Via PhysicsInformed Adaptive Moment Optimisation
}

\author{
Tongming $\mathrm{Qu}^{\mathrm{a}}$, Y.T. Feng ${ }^{\mathrm{a}}$, Min Wang ${ }^{\mathrm{b}}$, Shengqiang Jiang ${ }^{\mathrm{c}}$
}

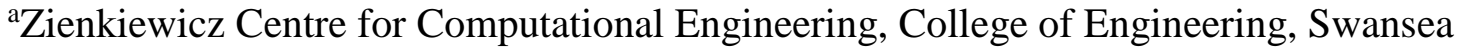

University, Swansea, Wales SA1 8EP, UK ${ }^{\mathrm{b}} \mathrm{T}-3$ Fluid Dynamics and Solid Mechanics Group, Theoretical Division, Los Alamos National

Laboratory, Los Alamos, New Mexico, 87545, USA 'School of Mechanical Engineering, Xiangtan University, Hunan, 411105, China

*Corresponding author; e-mail: y.feng@swansea.ac.uk 


\begin{abstract}
This study proposes an automated calibration procedure for bond parameters in bonded discrete element modelling. By exploring the underlying physical correlations between microscopic parameters of bonds and macroscopic strength parameters of the continuum to be modelled, the microscopic shear strength and tensile strength are identified as independent variables for calibration purpose. Then a physics-informed iterative scheme is proposed to automatically approximate the bond parameters by viewing the micro-macro relation as an implicitly defined mathematical mapping function. As a result of highly non-convex features of this implicit mapping, the adaptive moment estimation (Adam), which is especially suitable for problems with noisy gradients, is adopted as the basic iterative scheme, in conjunction with other numerical techniques to approximately evaluate the partial derivatives involved. The whole procedure offers a simple and effective framework for bond parameter calibration. A numerical example of $\mathrm{SiC}$ ceramic is provided for validation. By compared with some existing calibration methods, the proposed method shows significant advantages in terms of calibration efficiency and accuracy.
\end{abstract}

Keywords: discrete element method; parallel bond model; automated calibration; adaptive moment estimation; brittle solid; physics-informed optimisation

\title{
1 Introduction
}

The bonded particle model (BPM) is widely used to investigate the mechanical behaviour of solid materials, such as rock [1], concrete [2], ceramic [3], sea ice [4] and so on, because of the intrinsic ability of discrete element modelling in terms of directly reproducing the process of fracture in a continuum body. For continuum-based models, the properties used in the simulation can be derived directly from measurements performed on laboratory specimens. 
However, the input properties for BPM usually are not known in priori. How to efficiently choose reasonable particle-scale parameters to reflect the bulk behaviour of simulated materials is still an ongoing challenge.

The calibration of DEM parameters is a typical inverse problem. As a critical issue in the field of the discrete element method (DEM), calibration of particle-scale parameters receive extensive attentions. A large number of methods have been developed to address this challenge. Some design of experiments (DoE) methods, such as Taguchi methods [5], PlackettBurman(PB) and Central Composite Design (CCD) [6], are used to improve the try-and-error calibration process. The probability-based approach, such as the sequential quasi-Monte Carlo [7] and the Bayesian approach [8], are used for calibration purposes. The optimisation methods, such as the Genetic Algorithm (GA) [9, 10], Stable Noisy Optimisation by Branch and FIT (SNOBFIT) functions [11, 12], are used for calibration of bond parameters in bonded particle models. Furthermore, the most commonly reported method is the surrogate model. This model can be viewed as a "model of model" and utilises the data obtained by running the simulation model with different sets of parameters to approximate the relationship between inputs (particle-scale parameters) and outputs (macroscopic responses). Many different surrogate models, such as direct fitting method [13-15], polynomial response surface [16], artificial neural networks [17, 18], Kriging [19], random forest [20], Gaussian process regression and multi-adaptive regression-splines (MARS) [21], are utilised to calibrate DEM parameters. One challenge of these surrogate models is that the accuracy of the surrogate depends on the number and location of sampling points in the available parameter space, whereas the overall computational costs increase when a large number of computational models are required.

Although nearly all the above-mentioned methods are able to calibrate particle parameters in DEM, their practical applications are rather limited. Main reasons may be attributed to: (1) none of them is sufficiently efficient for an engineering application, as nearly hundreds of 
fullscale DEM simulations are often needed, and especially every single determination of strength parameters for a cemented material is computationally expensive; and (2) these methods normally require complex implementation processes and even involvements of multiple software, which becomes a barrier for many DEM users.

Recently, a hybrid analytical and computational framework has been developed by the authors $[22,23]$ to calibrate the particle-scale linear and non-linear deformation parameters within an accuracy of $1 \%$ or $2 \%$ after a few iterations. In the current paper, we extend this work to the calibration of parallel bond parameters with a physics-informed gradient-based optimisation method, aiming at addressing more complicated parameter calibration problems in DEM. It should be noted that many bonded particle models [1, 24-28] are available in DEM to model cohesive grain-based materials; however, this paper mainly focuses on the parameter calibration of the parallel bond model [1], which may be the most commonly used bonded particle model in DEM.

The current paper is divided into 5 sections. Following the introduction, Section 2 explores the relations between microscopic parameters and some macroscopic strength properties. Section 3 provides an automated computational framework to calibrate microscopic bond parameters based on both uniaxial compression test (UCT) and three-point bending test (TPBT). Section 4 presents an example to verify the reliability of the proposed framework. Section 5 discusses the calibration accuracy and the limitation of the current method. Section 6 offers some comprehensive remarks on the proposed methodology.

\section{Microscopic parameters and macroscopic strength for a bonded DEM specimen}

\subsection{A brief revisit of the parallel bond model}

The bonded particle, or discrete element, model is implemented by cementing two particles together at their contact points with a 'cement' (see Fig.1). The parameters that determine the 
mechanical behaviour of a bonded particle model are micro properties from both particles and the cement

- Particles (assuming the same material): Young's modulus of particles $E_{c}$, the ratio of shear to normal stiffness of the grains $\left(k_{s} / k_{n}\right)$, the friction coefficient of particles $(\mu)$.

- Cement: Young's modulus of the cement ${ }_{c}$, the ratio of shear to normal stiffness of the cement $\left(\begin{array}{c}k k_{s} /{ }_{n} \\ )\end{array}\right)$, the radius multiplier $\lambda$, the tensile strength ${ }_{\mathrm{c}}$ and the shear strength $\tau_{c}$.

The mechanical equations governing the force and displacement of both particles and cements can be referred to [1] for more detail. Specifically, the strength behaviour of a bonded particle model is mainly governed by the tensile strength and shear strength of the cement. Once the maximum tensile stress or maximum shear stress exceeds the tensile strength or shear strength of the cement, the parallel bond between the two particles breaks. At the same time, the accompanying force, moment and stiffness are removed from the model. The failure envelope of parallel bonds can be referred to Fig. 2. The shear strength is given by:

$$
\tau \bar{\sigma} \bar{\phi}_{\mathrm{c}}=-c_{\mathrm{n}} \overline{\tan }
$$

where $c$ and $\phi$ are the cohesion and friction angle of the parallel bond, respectively; and $\sigma_{\mathrm{n}}^{-}$is the normal stress at the bond periphery.

The above BPM is called the parallel bonded model as it can be envisioned as a set of elastic springs uniformly distributed over a rectangular cross-section (2D case) or a circular 
crosssection (3D case) lying on the contact plane and centred at the contact point. When the cement shrinks to a point (the size of the cement becomes zero), the BPM reduces to the contact bond model, which cannot resist a bending moment or oppose rolling; rather, it can only resist forces acting at the contact point. Due to the ability to transfer bending or twisting moment, many mechanical behaviours of the grain-based solid can be well reproduced by the

parallel bond model.

\subsection{Microscopic parameters associated with macroscopic strength properties}

Three sets of particle-scale parameters govern the mechanical responses of a bonded particle model. The influence parameters with the corresponding dimensions are listed in Table 1.

According to Buckingham's theorem [29], an arbitrary physical function $\phi\left(q_{1}, q_{2}, \ldots q_{n}\right)=0$, where $q_{i}$ is the $i^{t h}$ variable of $n$ physical variables, can be reformulated as $\phi\left(\pi_{1}, \pi_{2}, \ldots \pi_{n-k}\right)=0$, where $\pi_{i}$ is one of the dimensionless parameters, and $k$ is the number of independent physical units available.

In Table 1, 14 relevant parameters governing the macroscopic behaviour of a parallel bond model are listed. Because 3 independent physical units $[L, F, T]$ are used in a DEM model, 11 dimensionless parameters should be employed to describe the macroscopic behaviour. However, the selection of dimensionless parameters is not unique. For example, one possible dimensionless parameters set is: $\left[\mu, \phi, k_{s} / k_{n}, l / r, V E / \sqrt{\rho},_{s}^{k k_{n}, \phi,{ }_{c}} f_{c} e_{c} \tau I_{c}, \lambda_{,}^{-}-\right.$ $\left.E E_{c}{ }_{c}^{\prime}\right]$

The number of variables required to be considered can be further reduced due to the following reasons:

(1) In most simulations, the moduli and the ratios of normal to shear stiffness for both the particles and the cement are set to be the same value to reduce the number of free parameters 
$[1,30]$. For the case that $k_{n}$ and $k_{s}$ of the cement material are not the same as those of the particles, the proposed framework in our previous paper [22] still works. The difference is that two more independent parameters are required to update in the iterative process, the overall computational cost doubles and the calibration accuracy may slightly reduce due to a bigger challenge of convergence.

(2) This paper investigates how to calibrate the compressive strength and tensile strength of the bonded particle model. The calibration of elastic parameters (Young's modulus and Poisson's ratio) can refer to our previous work for linear contact model [22] and non-linear contact model [23]. Therefore, the particle-scale deformation parameters are not investigated in this study.

(3) Considering the physical roots of shear and tensile strength of the cemented material, it is reported that the $k_{s} / k_{n}$ ratio has measurable influences on the crack pattern and shear strength of DEM specimens [31]. Here we hold the opinion that the values of $k_{n}$ and $k_{s}$ are completely determined by the macroscopic Young's modulus and Poisson's ratio of the DEM specimen, rather than by the macroscopic shear strength.

(4) The existing research shows that the size dependence (specimen size/particle size) has a small influence on the compressive strength and tensile strength of a DEM specimen if the ratio $l / r$ is sufficiently large [30, 31].

(5) If numerical simulations are performed under quasi-static loading conditions, the loading velocity $V$ is no longer an influencing factor [32].

(6) The radius multiplier $\bar{\lambda}$ is used to set the length of the cement by multiplying $\bar{\lambda}$ to the smaller radius of two bonded balls. The calibration is performed under the condition that the cement completely fills the throat between the two boned particles in BPM so that the radius

multiplier $\overline{\lambda i s}$ set to be 1 . 
(7) The porosity $\phi$ and the density $\rho$ are two macroscopic indices to describe particle packings. The reported influence factors include the genesis pressure [1], the initial friction coefficient [22] and the mineral or material distribution [33]. The porosity $\phi$ and the density $\rho$ are important for determining the mechanical behaviour of a bonded particle model but these parameters cannot be directly inputted from simulations. In this paper, we restrict our calibration procedure to a model with a predetermined (but can be arbitrary, randomly distributed, polydisperse) packing configuration.

(8) Although the particle friction coefficient is an important strength parameter for determining instability of uncemented granular materials [34-36], its influence on the brittle failure is physically much more complex. To understand the role of friction in determining macroscopic strength properties, the failure mechanism of the bonded particle model is further explored below.

\subsection{Failure mechanism of a cemented specimen}

The bond parameters are usually calibrated by numerically performing some common strength experiments to make the numerical outputs agree with the experimental results. Normally, the UCT is used to calibrate the shear strength of a specimen by matching the experimental compressive strength. The tensile strength of a specimen can be calibrated by matching the flexural strength in TPBT or matching the tensile strength in brazil discs tests (BDT). Considering the complexities of stress distribution and loading configurations in BDT [37], both UCT and TPBT models are used in this study as benchmark experiments to calibrate the shear bond and tensile bond parameters.

Understanding the physics behind a failure phenomenon may be helpful for more effectively calibrating the microscopic parameters. The macroscopic failure is attributed to microscopic failures, especially in the form of either tensile failure or shear failure in the bonded particle 
model [38]. For a cemented specimen subjected to uniaxial compression testing, its overall failure is normally characterised by three stages (see Fig. 3):

Stage I is a completely elastic process prior to any contact bond failure. In this stage, the deformation is completely recoverable and the influence of the friction coefficient can be ignored.

Stage II is signified by the onset of particle-scale cracking (bond breaks), in which the cohesion of the whole specimen is weakened (bonds between grains break gradually) and the frictional strength plays increasing influences due to progressive contact slidings involved (frictional strengthening) [39, 40]. Although local failure occurs in this stage, the whole specimen has not reached a complete failure until the maximum stress that the specimen can withstand is reached. This transition from continuum to dis-continuum behaviour is a failure sign of the bonded particle model from a brittle to a hybrid brittle and ductile behaviour. Irreversible deformation happens inside the specimen during this stage.

Stage III is a post-peak state where the compressive stress of the specimen passes through the maximum stress and declines gradually until a rupture of the whole specimen occurs. The frictional strengthing and cohesion weakening continues during this stage. From brittle failure (featured as cracks) to ductile failure (frictional strength, featured as a shear band or strain localized area), this is a sign that the system gradually evolves toward a granular state.

For a TPBT model, Fig. 4 shows the specimen that undergoes the maximum bending moment at the central line of the specimen. The specimen between the two supporting points bears similar shear forces. Therefore, the bottom and top points at the central line of the specimen are the most likely locations undergoing compression-induced failure and tensile failure for the perspective of loading.

Table 2 summarises the ${ }_{\mathrm{c}}^{\sigma} l_{\mathrm{c}}^{\tau}$ ratios for commonly simulated grain-based materials. It can 
be seen that the tensile bond strength is normally less than the shear bond strength. Considering the load condition in a TPBT model, the flexural strength is mainly determined by the tensile

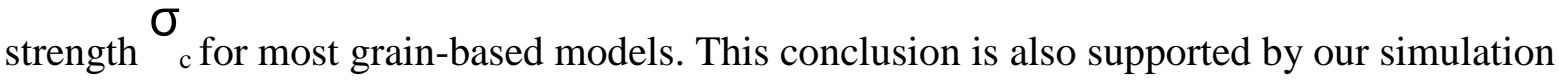
results.
}

The failure of a TPBT model is initiated from some tensile cracks at the bottom position around the central line of the specimen. Once a crack or deterioration happens, other cracks will develop one after another until the loading ceases to be applied. In this process, the particle friction plays a very limited role.

To investigate the role of particle-scale friction on the macroscopic strengths, a series of TPBT and UCT simulations with different coefficients of friction between particles are performed (the same packing configuration is used to eliminate the influences of the granular fabric structure). The parameters used in the simulation can be found in Table 3. To facilitate the comparison, the strength values are normalised with the corresponding compressive or flexural strength determined from the cases with zero particle friction.

Our numerical results in Fig. 5 show that a friction coefficient of 1.0 may strengthen the final compressive strength by around $12 \%$ and strengthen the final flexural strength by about $4 \%$, compared to the specimen with no friction. It could be concluded that the particle friction has limited influences on both uniaxial compressive strength and flexural strength. A similar conclusion is also reported in [1, 11, 32, 46]. Wang and Tonon [11] found that a measurable effect of the friction angle on the material strength can be observed only when the cohesion is relatively low but the overall influence is still limited.

Considering the complex mechanism involved and the limited influence of particle-scale on the overall strength of the specimen, a friction coefficient of 0.5 is recommended as a reasonable value for the simulation of a bonded particle model [1]. Now the primary 
microscopic parameters for determining the compressive and tensile strength of a contactbonded specimen are thus reduced to ${ }_{c}$ and ${ }_{c}^{\tau}$.

\section{An automated calibration procedure for bond parameters}

In DEM models, the microscopic parameters are direct inputs, while the simulated macroscopic properties are the emerging outputs of the system. It bears resemblance to a mathematical equation, where the independent variables are inputs and dependent variables are outputs. By viewing the calibration of DEM parameters as a process of solving an equation, an iterative scheme can be developed for the purpose of calibrating parameters. This procedure includes the following steps: (1) construct an objective function incorporating calibration targets; (2) determine an iterative scheme suitable for calibration problems of bond parameters; and (3) derive technical details including feature scaling, gradient approximation, and initial estimations of bond parameters. These steps will be individually discussed in detail in Sections 3.1-3.5. A physics-informed simplification is proposed in Section 3.6 and the overall workflow of the calibration procedure is given in Section 3.7.

\subsection{Constructing an objective function}

The calibration of bond parameters is conceptually equivalent to finding roots for an equation:

$$
f_{D E M}\left(\overline{\sigma \tau}, \bar{c},{ }_{c}\right)-f_{\text {experiment }}=0
$$

When the exact solutions of the equation are not available, the calibration problem is converted to a problem of finding approximate solutions or to a minimisation problem. For the current 
problem concerned, an objective function, that combines the calibrations of both uniaxial compressive strength and flexural strength together, is given as follows:

$$
L\left(\sigma \tau_{c}, \bar{c}\right)=\lambda \sigma \sigma_{1}(\mathrm{cct}-1)+{ }^{2} \lambda \sigma \sigma_{2}(\quad f f t-1)^{2}
$$

where $\sigma_{\mathrm{c}}$ and $\sigma_{f}$ are the uniaxial compressive strength and flexural strength to be determined

for a bonded particle model; $\sigma_{c t}$ and $\sigma_{f t}$ are the targeted compressive strength and flexural strength, respectively; $\lambda_{1}$ and $\lambda_{2}$ are two positive weighting coefficients with $\lambda_{1}+\lambda_{2}=1$. Different combinations of $\lambda_{1}$ and $\lambda_{2}$ can lead to placing different degrees of emphasis on $\sigma_{\mathrm{c}}$ and $\sigma_{f}$. When both compressive strength and flexural strength are to be calibrated with equal importance, $\lambda_{1}=\lambda_{2}=0.5$.

\subsection{The iterative algorithm}

Many iterative algorithms, such as the Newton-Raphson Method, Gradient descent methods etc., are able to solve a nonlinear equation. However, the calibration problem has its own special features: (1) objective functions in calibration problems cannot be explicitly expressed (no analytical forms available) and are usually discontinuous; (2) The grain-based bond specimen is highly heterogeneous [47] and the relationship between the microscopic bond parameters and the macroscopic strength is a non-convex function. To address the first issue, a finite-difference based scheme is used to approximate the gradient of the objective function [48]. It is computationally expensive to use the difference method to approximate a secondorder derivative, thus the Newton-Raphson method is not a good choice. To address the second issue, the adaptive moment estimation (Adam) algorithm $[49,50]$, which works well in problems with very noisy gradients (non-convex function), are applied in this study to calibrate the bond parameters. 
Adam is a first-order gradient-based optimisation algorithm. The implementation of Adam requires computing an exponentially decaying average of past gradients (the first moment, say $M_{t}$ ) and an exponentially decaying average of past squared gradients (the second moment, say $G_{t}$ ) as follows:

$$
\begin{gathered}
M_{t}=\beta_{1} M_{t-1}+-\left(1 \beta_{1}\right) g_{t} \\
G_{t}=\beta_{2} G_{t-1}+-\left(1 \beta_{2}\right) g_{t} \otimes g_{t}
\end{gathered}
$$

where $g_{t}$ denotes the gradient of the objective function at the $t^{\text {th }}$ iterative step; the subscript $t$ represents the iterative number (starting from 1); the symbol $\otimes$ represents element-wise multiplication; $\beta_{1}$ and $\beta_{2}$ are the decay rates of the first-moment and the second-moment of the gradient; and their values are typically initialised to be 0.9 and 0.99 , respectively.

The moment estimates (both $M_{t}$ and $G_{t}$ ) are biased towards zero at the initial timesteps and especially when both decay rates are close to 1 [49] because the moving averages are initialised as zero vectors. This initialisation bias is practically corrected with bias-corrected moment estimates as follows:

$$
\begin{gathered}
M_{t}=\frac{M_{t t}}{1-\beta_{1}} \\
G^{\wedge}={ }_{1-\beta_{2}} G_{t}
\end{gathered}
$$

To find solutions or the minimum of a function using the Adam algorithm, independent variables involved (say $\theta_{i}$ ) are iteratively updated by:

$$
\theta \theta_{i}:=\begin{gathered}
\alpha^{\wedge} \\
\sqrt{G^{\wedge}+\varepsilon}
\end{gathered}
$$

where the symbol ":=" means assignment; the symbol $\varepsilon$ is a smoothing term usually initialised to a tiny number such as $10^{-8}$; and $\alpha$ is the learning rate. The selections of $\alpha$ will affect the 
iterative process (including the convergent rate and accuracy). Empirically, the learning rate can be chosen from $0.1,0.01$, and 0.001 to see their influences on the optimisation process.

To facilitate the convergence of iterations, the value of the objevtive or cost function $L$ is used to multiply the original increments in each iteration, as follows:

$$
\theta \theta_{i}:=\begin{gathered}
\alpha L^{\wedge} \\
\sqrt{{G^{\prime}}_{t}+\varepsilon}
\end{gathered}
$$

The reasons of making such a multiplication are: 1) the iterative increments will be downscaled to reduce the risk of the overshoot problem when the iterative predictions are near the calibration target (the value of $L$ will converge towards 0 ); and 2) the iterative increment will be upscaled to accelerate the converge if the current iterative values are far from the calibration target (the value of $L$ is larger than 1.0).

\subsection{Feature scaling}

Since the range of a microscopic bond strength may vary widely, data normalisation or feature scaling is necessary to facilitate the converge of the iterative process. In this study, both

microscopic shear bond strength ${ }_{\mathrm{c}}^{-}$and tensile bond strength ${ }_{\mathrm{c}}$ are normalised by the corresponding targeted compressive strength $\sigma_{\text {ct }}$ and flexural strength ${ }_{f t}$, and the resulting normalised microscopic shear bond strength $\tau_{\mathrm{c}}$ and tensile bond strength $\sigma_{\mathrm{c}}$ are denoted as:

$$
\begin{array}{cc}
\overline{\mathcal{I}} & \bar{\sigma} \\
\tau^{\wedge}{ }_{c}={ }^{c}, \sigma^{\wedge}{ }_{c}= & { }^{c} \\
\sigma_{c t} & \sigma_{f t}
\end{array}
$$

Then the objective function can be rewritten as:

$$
L\left(\sigma \tau \bar{\sigma} \tau_{\mathrm{c}}, \mathrm{c}\right)=\left(F_{\hat{c}}, \hat{c}\right)
$$




\subsection{Gradient approximation}

Although the closed-form relationship between the microscale bond parameters and the macroscopic strength parameters are not known, the gradient of the objective function can still be approximated by using the finite-difference method. Let the gradient of the objective function, $g_{t}$, be:

$$
=\frac{\partial F}{\partial^{\wedge}} \frac{\partial F}{\partial^{\wedge}} g_{t} \quad(\quad, \quad) \quad \text { (12) } \sigma \quad \tau_{c}
$$

Then the partial derivatives of the objective function can be approximated as:

$$
\begin{aligned}
& \square \partial F \sigma \\
& \mathrm{z} \approx_{f t}\left[L\left(\sigma \sigma \tau_{c}+\Delta_{c},{ }_{c}\right)-L\left(\sigma \tau_{c},{ }_{c}\right)\right] \\
& \square \partial \sigma \sigma^{\wedge}{ }_{\Lambda_{-}} \quad-\quad--C_{-} \\
& \square
\end{aligned}
$$

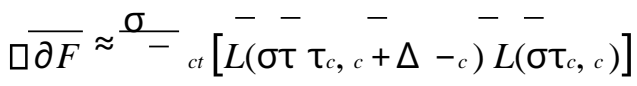

$$
\begin{aligned}
& \operatorname{Do} \partial \tau \tau^{{ }_{c}} \Delta_{c}
\end{aligned}
$$

where $\Delta^{-}{ }_{c}$ and $\Delta^{\tau}{ }_{c}$ are two (small) increments of the microscopic bond parameters. The computations of the partial derivatives in the above procedure can be carried out independently, and therefore can be fully parallelised.

\subsection{Initial estimations}

Good initial approximations are important to reduce the risk of falling into the trap of a local optimum and to facilitate obtaining a global optimum during the iterations. Although the microscopic bond strength is different from the macroscopic strength, some underlying connections between them exist: (i) The contact-scale tensile or shear failure does not mean the failure of the whole specimen, but the macroscopic failure is fundamentally caused by microscopic bond failures; (ii) In the case that all the cement strengths are the same, the bond 
failure starts from the cement undergoing a larger contact force. Although the contact forces between particles are different from each other because the granular assembly transfers external loads in force chains [51-55], the magnitude of the contact force for most contacts is distributed within a narrow range [56]; and (iii) Not rigorously verified though, the stress experienced by the cement is similar to a stress component perpendicular to the contact plane, from the perspective of continuum mechanics. All the underlying connections may explain that the differences between the contact-scale bond strength and the macroscopic strength are limited in the literature $[1,3]$.

In this study, the macroscopic strength parameters determined from laboratory experiments are used as a starting point for the calibration of the particle-scale bond parameters, namely:

$$
\tau \sigma \sigma \sigma_{c}:={ }_{c t}
$$

The same initial estimation is used in $[13,45]$. The research in [14] also shows that a linear relationship between the macroscopic tensile strength and the microscopic tensile strength under the conditions that all the packing parameters and deformation parameters are kept the same. Furthermore, larger microscopic bond parameters intuitively will give rise to larger macroscopic strengths. Inspired by this idea and the parametric analysis conducted in [14], the second estimation of microscopic parameters are developed as follows:

$$
\begin{gathered}
\bar{\tau}_{c}:=\sigma_{c t} \bar{\tau} \sigma_{c} \bar{c}_{c}:=\overline{\sigma_{f t}} \overline{\sigma_{c}} \\
\sigma_{c} \quad \sigma_{f}
\end{gathered}
$$

After determining the first and second estimations of the microscopic parameters, the gradient of the objective function can be approximated by Eq.(13). The microscopic bond parameters can be iteratively updated by Eq.(9) until a satisfactory calibration accuracy is achieved. 
3.6 Possible simplification by considering more underlying physics mechanisms

From the perspective of continuum mechanics, the uniaxial compressive strength determined from uniaxial compressive tests (UCT) is due to the shear failure of the specimen, while the tensile failure dominates the flexural strength in three-point bending tests (TPBT). We thus infer

that

$$
\mathrm{UCT}\left(\sigma \sigma \tau \tau_{c}+\Delta{ }_{c},{ }_{c}+\Delta \approx_{c}\right) \mathrm{UCT}\left(\sigma \tau \tau_{c},{ }_{c}+\Delta_{c}\right)
$$

$$
\text { the } \quad \operatorname{TPBT}\left(\sigma \tau \tau_{c},{ }_{c}+\Delta_{c}\right) \approx \operatorname{TPBT}\left(\sigma \bar{\tau}_{c},{ }_{c}\right)
$$

$$
\operatorname{TPBT}\left(\sigma \overline{\sigma \tau} \tau_{c}+\bar{\Delta}_{c, c}+\Delta \approx_{c}\right) \operatorname{TPBT}\left(\sigma \bar{\sigma} \tau_{c}+\Delta{ }_{c}, c\right)
$$

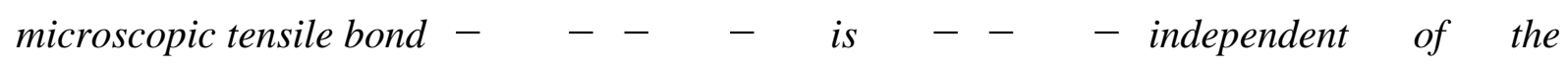
compressive strength in UCT and that the shear bond is irrelevant to the flexural strength in $T P B T$. The procedure to find the value of the objective function can thus be simplified as follows:

$$
\mathrm{UCT}\left(\sigma \bar{\sigma}_{c}+\bar{\Delta}_{c}, c\right) \approx \mathrm{UCT}\left(\bar{\sigma} \bar{\tau}_{c}, c\right)
$$

where $\mathrm{UCT}()$ and TPBT () represent the compressive strength obtained from the UCT and the flexural strength obtained from the TPBT with the input parameters in brackets.

The original iterative procedure requires 3 sets of full DEM simulations at each iteration (3 UCT and 3 TPBT). By using the above simplification, only one set of simulations (1 UCT and 1 TPBT) is needed. Thus the computational cost can be reduced by three times. Considering the fact that the microscopic $\bar{\sigma}_{\mathrm{c}} / \bar{\tau}_{\mathrm{c}}$ ratios in the modelling of common brittle materials are less than 0.8 (see Table 2), such a simplification scheme is practically useful.

The problem of this simplified method is that the particle-scale bond failure is highly complex compared to the continuum due to heterogeneous features in a grain-based model and thus the foundation of making such a simplification may not be rigorous. A number of 
numerical tests were performed to evaluate the relations between the micro bond strength and macro compressive strength. The micro shear bond strength basically determines the

compressive strength in UCT for the model with a relatively low $\bar{\sigma}_{\mathrm{c}} / \bar{\tau}_{\mathrm{c}}$ ratio (less than 1.0), while both shear failure and tensile failure affect the compressive strength of the specimen (both shear failure and tensile failure exist) for the bonded particle model with a high $\sigma_{\mathrm{c}} / \bar{\tau}_{\mathrm{c}}$ ratio (larger than 1.0). For the models with the $\bar{\sigma}_{\mathrm{c}} / \bar{\tau}_{\mathrm{c}}$ ratio around 1.0, the random packing structure in a DEM model may affect the microscopic failure mode. For the sake of rigorousness, here we restrict the use of this simplified scheme to the bonded particle model

with a relatively low $\sigma_{\mathrm{c}} / \tau_{\mathrm{c}}$ ratio (lower than 0.8 ). In contrast, the original scheme is valid for the bonded particle models with any $\sigma_{\mathrm{c}} / \bar{\tau}_{\mathrm{c}}$ ratio.

\subsection{The workflow of the proposed calibration methodology}

The proposed framework to conduct a standard calibration procedure is displayed in Fig. 6. The first step is to obtain the calibration target and is normally completed by performing laboratory experiments of TPBT and UCT. After determining the flexural strength and compressive strength of the simulated specimen, a set of initial approximations on the selected microscopic bond parameters is made (Eq.(14)). By performing numerical TPBT and UCT, the second estimation is made on the basis of the numerical flexural strength and compressive strength (Eq.(15)). By performing numerical TPBT and UCT with the second estimation, the microscopic bond parameters can be iteratively updated following Eq.(4)-(13). A check on the value of the objective function is required at each step, and the iterative process is performed 
until the predefined accuracy is achieved. A detailed discussion about the tolerance (or calibration accuracy) is given in Section 5.

\section{Numerical verification}

A 2D case of calibrating a bonded discrete model of SiC ceramics is used to verify the ability of the proposed calibration framework. The flexural strength in TPBT and the compressive strength in UCT are experimentally determined as the calibrating targets: the flexural strength is $0.78 \mathrm{GPa}$ and the compressive strength is $2 \mathrm{GPa}$. The TPBT and UCT models (see Fig. 7) include 22507 and 66743 disc particles, respectively. As the purpose of this study is to calibrate bond parameters, the deformation parameters and model size reported in $[57,58]$ are directly adopted. The calibration methods for deformation parameters can be found in our recent work $[22,23]$.

By following the calibration procedures summarised in Section 3.5, both the original scheme and the simplified scheme are performed. A learning rate of 0.01 is used in both schemes. As Fig. 8 shows, both schemes are able to obtain a reasonable estimation in a few iterations. The original version slightly outperforms the simplified version in terms of the accuracy achieved. The best prediction accuracy of the original version reached in 6 iterations is around $2.7 \%$ for the flexural strength in TPBT and $1.6 \%$ for the compressive strength in UCT. The corresponding best prediction accuracy that the simplified version can achieve in 6 iterations is around $1.5 \%$ for the flexural strength in TPBT and 3\% for the compressive strength in UCT (in the third iteration step). The microscopic bond parameters at each iterative step are given in Table 4 for the original version and in Table 5 for the simplified version. This case shows that the proposed procedure is able to calibrate the microscopic bond parameters in a few iterations. 


\section{Discussion}

5.1 The calibration efficiency and accuracy

5.1.1 A comparison between the proposed methodology and other reported calibration methods A summary of calibration efficiency and accuracy of microscopic bond parameters is given in Table 6. Considering the fact that each calibration method is conducted in various DEM models with different particle numbers and loading conditions, the calibration efficiency of each method is evaluated with the number of numerical experiments required. The calibration performance of our proposed method is also added for comparison. The results demonstrate that the proposed physics-informed adaptive moment algorithm (Adam) (both the original and simplified iteration schemes) outperforms existing calibration methods in terms of calibration accuracy and efficiency. Furthermore, the proposed algorithm has strong adaptability to the specimen with varied fabric configurations whereas surrogate-based models may lose some accuracy when predicting the microscopic parameters in models beyond existing experiences.

\subsubsection{The acceptable level of calibration accuracy}

For a closed-form equation, numerical methods can obtain a high-accuracy solution, but it is not the case for the calibration problem. The accuracy of parameter calibration in DEM may be affected by the following factors:

(1) high heterogeneity in a grain-based system

In bonded particle models, a failure either in tension or shear form is generally initiated from those highly stressed contact bonds and then propagates gradually. The heterogeneity may lead to some local small defects within materials (such as the area with a relatively large void). Local failures usually start and develop around these initial defects, and thus the recorded strength values are highly dependent on the conditions of these defects. Furthermore, high heterogeneity in a grain-based system may cause high-stress intensity at some local areas. These features give rise to a significant non-convex relationship between the macroscopic 
strength and microscopic bond parameters and thus similar sets of the microscopic parameters may produce the macroscopic responses within a stochastic fluctuation range.

(2) slight packing differences between TPBT and UCT models

The bond parameters are calibrated with TPBT and UCT models at the same time; however, inevitable inconsistency exists in these two models in terms of the packing structure. A set of microscopic parameters suitable for a TPBT model may not perform well in a UCT model. The final microscopic parameters are required to enable the two different models to match the respective experiment well. A large difference between the packing structures in the two models may deteriorate the final calibration precision.

Considering the inherent features of the grain-based model, to achieve a perfect calibration match is not realistic. Then what level of calibration accuracy is acceptable in the practical term? Actually, the calibration target, namely experimental results, also suffer from typical experimental scatters. Wang and Tonon [11] choose a tolerance criterion at 5\%. Wang and Cao [59] and Chen et al [61] set the maximum error to be 10\%. Based on the authors' calibration and experimental experiences, an accuracy of 5\% may be reasonable due to the scattering nature of experimental measurements. Also, it is unnecessary to calibrate the bond parameters meticulously. Furthermore, an accuracy of $1 \%$ is very hard to be achieved due to a fluctuation of the system and the packing difference between the two models.

5.2 The ratio of compressive strength $\left(\sigma_{c}\right)$ to tensile strength $\left(\sigma_{t}\right)$

The proposed calibration procedure works well in the verification case. However, it seems to have an intrinsic limitation in calibrating a bonded particle model for a high $\sigma \sigma_{c} /{ }_{t}$ ratio material such as brittle rocks [1]. The reasons may be attributed to the limitation of sphere or circular elements in DEM models in reproducing significant grain interlocking in low-porosity hard rocks [63]. 
A simple but useful remedial measure is to generate a slightly overlapped particle configuration with a higher genesis pressure $[31,64]$. The genesis pressure $\left(\sigma_{o}\right)$ tends to be used during specimen preparation and is applied to the specimen before particle bonds are added. The main purpose of such a genesis pressure is to compact the granular packing before adding bonds between particles. Potyondy and Cundall [1] initially reported that a suitable selection of the genesis pressure is helpful to reduce the magnitude of the locked-in forces that will develop after the parallel bonds are added. Later on, the genesis pressure is found to be related to the mechanical response of cemented materials. A relatively large genesis pressure can be used to create a small contact overlap between disks. This small overlap is helpful for obtaining a more realistic friction angle and a ratio of compressive to tensile strength of the simulated DEM material [64]. It is also found that the use of a high genesis pressure enables a more realistic failure envelope and tensile strength to be simulated, while it was not possible previously by using a circular element with zero genesis pressure [31].

Apart from the slightly overlapped particle model, many other methods are also employed to overcome the shortcomings of a circular or spherical particle configuration, such as particle clusters [1,33], rigid particle clumps [65-67], stochastic distribution of material properties [68], other bonded particle model [69], and polygonal particles [70].

The slightly overlapped particle model cannot be directly determined by using the input parameters in DEM and it is also not clear what overlapping degree is sufficient to achieve a certain $\sigma \sigma /_{c}$ ratio. Furthermore, the methods of cluster or clump particles may suffer from a disagreement between the simulated size and the real size distribution of constituent components [15]. The current study aims to provide calibration procedures for particle or discsbased parallel bond models, and thus other remedial measures are not considered here.

The current calibration procedure, therefore, will be restricted to its predictive capability for cemented materials with a low $\sigma \sigma_{c} /{ }_{t}$ ratio (typically 3-5), such as ceramics and weak cemented 
soils or rocks. Automatically calibrating bond parameters with a capacity of reproducing any material with a realistically high $\sigma \sigma_{c} /{ }_{t}$ ratio is out of the scope of this study but will be explored in the future.

\section{Conclusion remarks}

An automated computational methodology has been proposed to calibrate the bond parameters in the parallel bond model in DEM. The possible microscopic factors associated with macroscopic strength parameters have been investigated first with the dimension analysis method. On the basis of underlying physical relations between microscale bond parameters and macroscopic strengths of the material, the first and second estimations of both shear bond and tensile bond parameters are given. After performing the UCT and TPBT models with the improved Adam algorithm in a few iterative steps, the approximation of bond parameters is proven to be able to match the predefined macroscopic bond parameters satisfactorily for the bonded particle model with a low $\sigma \sigma_{c} /{ }_{t}$ ratio. A comparison between the proposed method and existing methods shows that the proposed methodology is able to achieve a higher calibration accuracy with fewer iterations.

The success of the proposed calibration procedure can be attributed to two factors: (i) the underlying physics relationships between the microscopic and macroscopic parameters are fully exploited and used. This is the fundamental reason that the proposed calibration procedure is effective; and (ii) the proposed iterative algorithm is particularly suitable for problems with noisy gradients. This exactly matches the inherent features of calibration problems in DEM.

Although the proposed methodology, in principle, can be applied to calibration problems of other bonded particle models, more numerical investigation with specified BPMs and specific materials are required before some concrete conclusions can be drawn. 
In the present study, we assume that the grain-based cemented material is composed of only one material type and one bond strength. This is not the general case as many cemented materials, such as rocks, often consist of several components. In our future work, a multicomponent bonded particle model will be explored. Furthermore, a framework aiming to calibrate the bond parameters with a capacity of reproducing any material with a realistically high $\sigma \sigma_{c} /{ }_{t}$ ratio will also be developed.

\section{References}

[1] D.O. Potyondy, P.A. Cundall, A bonded-particle model for rock, International Journal of Rock Mechanics and Mining Sciences, 41 (2004) 1329-1364.

[2] M. Nitka, J. Tejchman, Modelling of concrete behaviour in uniaxial compression and tension with DEM, Granular Matter, 17 (2015) 145-164.

[3] Y. Tan, D. Yang, Y. Sheng, Discrete element method (DEM) modeling of fracture and damage in the machining process of polycrystalline $\mathrm{SiC}$, Journal of the European ceramic society, 29 (2009) 1029-1037.

[4] S. Ji, S. Di, X. Long, DEM simulation of uniaxial compressive and flexural strength of sea ice: parametric study, Journal of Engineering Mechanics, 143 (2016) C4016010.

[5] K.J. Hanley, C. O'Sullivan, J.C. Oliveira, K. Cronin, E.P. Byrne, Application of Taguchi methods to DEM calibration of bonded agglomerates, Powder technology, 210 (2011) 230240.

[6] J. Yoon, Application of experimental design and optimization to PFC model calibration in uniaxial compression simulation, International Journal of Rock Mechanics and Mining Sciences, 44 (2007) 871-889.

[7] H. Cheng, T. Shuku, K. Thoeni, H. Yamamoto, Probabilistic calibration of discrete element simulations using the sequential quasi-Monte Carlo filter, Granular matter, 20 (2018) 1-19.

[8] H. Cheng, T. Shuku, K. Thoeni, P. Tempone, S. Luding, V. Magnanimo, An iterative

Bayesian filtering framework for fast and automated calibration of DEM models, Computer

Methods in Applied Mechanics and Engineering, 350 (2019) 268-294.

[9] J. Pachón-Morales, H. Do, J. Colin, F. Puel, P. Perré, D. Schott, DEM modelling for flow of cohesive lignocellulosic biomass powders: Model calibration using bulk tests, Adv Powder Technol, 30 (2019) 732-750.

[10] H.Q. Do, A.M. Aragón, D.L. Schott, A calibration framework for discrete element model parameters using genetic algorithms, Adv Powder Technol, 29 (2018) 1393-1403.

[11] Y. Wang, F. Tonon, Calibration of a discrete element model for intact rock up to its peak strength, International journal for numerical and analytical methods in geomechanics, 34 (2010) 447-469.

[12] Y. Wang, F. Tonon, Modeling Lac du Bonnet granite using a discrete element model, International Journal of Rock Mechanics and Mining Sciences, 46 (2009) 1124-1135. 
[13] B.S.A. Tatone, G. Grasselli, A calibration procedure for two-dimensional laboratoryscale hybrid finite-discrete element simulations, International Journal of Rock Mechanics and Mining Sciences, 75 (2015) 56-72.

[14] T.-T. Nguyen, D. André, M. Huger, Analytic laws for direct calibration of discrete element modeling of brittle elastic media using cohesive beam model, Computational Particle Mechanics, 6 (2019) 393-409.

[15] T. Kazerani, J. Zhao, Micromechanical parameters in bonded particle method for modelling of brittle material failure, International journal for numerical and analytical methods in geomechanics, 34 (2010) 1877-1895.

[16] S. Chehreghani, M. Noaparast, B. Rezai, S.Z. Shafaei, Bonded-particle model calibration using response surface methodology, Particuology, 32 (2017) 141-152.

[17] L. Benvenuti, C. Kloss, S. Pirker, Identification of DEM simulation parameters by

Artificial Neural Networks and bulk experiments, Powder technology, 291 (2016) 456-465.

[18] H. Zhou, Z. Hu, J. Chen, X. Lv, N. Xie, Calibration of DEM models for irregular particles based on experimental design method and bulk experiments, Powder Technology, 332 (2018) 210-223.

[19] M. Rackl, K.J. Hanley, A methodical calibration procedure for discrete element models, Powder technology, 307 (2017) 73-83.

[20] A. Boikov, R. Savelev, V. Payor, DEM Calibration Approach: Random Forest, Journal of Physics: Conference Series, IOP Publishing, 2018, pp. 012009.

[21] C. Richter, T. Rößler, G. Kunze, A. Katterfeld, F. Will, Development of a standard calibration procedure for the DEM parameters of cohesionless bulk materials-Part II: Efficient optimization-based calibration, Powder Technology, 360 (2019) 967-976.

[22] T. Qu, Y. Feng, T. Zhao, M. Wang, Calibration of Linear Contact Stiffnesses in Discrete Element Models Using a Hybrid Analytical-Computational Framework, Powder Technology, 356 (2019) 795-807.

[23] T. Qu, Y. Feng, T. Zhao, M. Wang, A hybrid calibration approach to Hertz-type contact parameters for discrete element models, International Journal for Numerical and Analytical Methods in Geomechanics, (2020) In press.

[24] D. Potyondy, A flat-jointed bonded-particle material for hard rock, 46th US rock mechanics/geomechanics symposium, American Rock Mechanics Association, 2012.

[25] M. Obermayr, K. Dressler, C. Vrettos, P. Eberhard, A bonded-particle model for cemented sand, Computers and Geotechnics, 49 (2013) 299-313.

[26] N.J. Brown, J.-F. Chen, J.Y. Ooi, A bond model for DEM simulation of cementitious materials and deformable structures, Granular Matter, 16 (2014) 299-311.

[27] M. Jiang, F. Zhu, F. Liu, S. Utili, A bond contact model for methane hydrate-bearing sediments with interparticle cementation, International Journal for Numerical and Analytical Methods in Geomechanics, 38 (2014) 1823-1854.

[28] Z. Shen, M. Jiang, C. Thornton, DEM simulation of bonded granular material. Part I: contact model and application to cemented sand, Computers and Geotechnics, 75 (2016) 192-209.

[29] E. Buckingham, On physically similar systems; illustrations of the use of dimensional equations, Physical review, 4 (1914) 345-376.

[30] B. Yang, Y. Jiao, S. Lei, A study on the effects of microparameters on macroproperties for specimens created by bonded particles, Engineering Computations, 23 (2006) 607631. 
[31] A. Fakhimi, T. Villegas, Application of dimensional analysis in calibration of a discrete element model for rock deformation and fracture, Rock Mechanics and Rock Engineering, 40 (2007) 193.

[32] X. He, C. Xu, Discrete element modelling of rock cutting: from ductile to brittle transition, International Journal for Numerical and Analytical Methods in Geomechanics, 39 (2015) 1331-1351.

[33] C. Shi, W. Yang, J. Yang, X. Chen, Calibration of micro-scaled mechanical parameters of granite based on a bonded-particle model with 2D particle flow code, Granular Matter, 21 (2019) 1-13.

[34] J.R. Rice, The localization of plastic deformation, in: W.T. Koiter (Ed.) the 14th

International Congress on Theoretical and Applied Mechanics, North Holland Publishing Co., Delft, 1976, pp. 207-220.

[35] T. Qu, S. Wang, Q. Hu, Coupled Discrete Element-Finite Difference Method for Analysing

Effects of Cohesionless Soil Conditioning on Tunneling Behaviour of EPB Shield, KSCE Journal of Civil Engineering, 23 (2019) 4537-4551.

[36] T. Qu, S. Wang, J. Fu, Q. Hu, X. Zhang, Numerical Examination of EPB Shield Tunneling-

Induced Responses at Various Discharge Ratios, J Perform Constr Fac, 33 (2019) 04019035.

[37] D. Li, L.N.Y. Wong, The Brazilian disc test for rock mechanics applications: review and new insights, Rock mechanics and rock engineering, 46 (2013) 269-287.

[38] M. Xia, K.-p. Zhou, Particle simulation of the failure process of brittle rock under triaxial compression, International Journal of Minerals, Metallurgy, and Materials, 17 (2010) 507513.

[39] V. Hajiabdolmajid, P. Kaiser, C. Martin, Modelling brittle failure of rock, International Journal of Rock Mechanics and Mining Sciences, 39 (2002) 731-741.

[40] V. Hajiabdolmajid, P. Kaiser, C. Martin, Mobilised strength components in brittle failure of rock, Geotechnique, 53 (2003) 327-336.

[41] K. Duan, C. Kwok, L. Tham, Micromechanical analysis of the failure process of brittle rock, International Journal for Numerical and Analytical Methods in Geomechanics, 39 (2015) 618-634.

[42] S.-Q. Yang, Y.-H. Huang, H.-W. Jing, X.-R. Liu, Discrete element modeling on fracture coalescence behavior of red sandstone containing two unparallel fissures under uniaxial compression, Engineering Geology, 178 (2014) 28-48.

[43] S.-Q. Yang, Y.-H. Huang, P. Ranjith, Y.-Y. Jiao, J. Ji, Discrete element modeling on the crack evolution behavior of brittle sandstone containing three fissures under uniaxial compression, Acta Mechanica Sinica, 31 (2015) 871-889.

[44] S. Zhou, H. Zhu, Z. Yan, J.W. Ju, L. Zhang, A micromechanical study of the breakage mechanism of microcapsules in concrete using PFC2D, Construction and Building Materials, 115 (2016) 452-463.

[45] E. Oñate, F. Zárate, J. Miquel, M. Santasusana, M.A. Celigueta, F. Arrufat, R. Gandikota, K. Valiullin, L. Ring, A local constitutive model for the discrete element method. Application to geomaterials and concrete, Computational particle mechanics, 2 (2015) 139160. 
[46] H.-y. Huang, B. Lecampion, E. Detournay, Discrete element modeling of tool-rock interaction I: rock cutting, International Journal for Numerical and Analytical Methods in Geomechanics, 37 (2013) 1913-1929.

[47] S. Liakas, C. O'Sullivan, C. Saroglou, Influence of heterogeneity on rock strength and stiffness using discrete element method and parallel bond model, Journal of Rock Mechanics and Geotechnical Engineering, 9 (2017) 575-584.

[48] Y. Feng, T. Zhao, J. Kato, W. Zhou, Towards stochastic discrete element modelling of spherical particles with surface roughness: A normal interaction law, Computer Methods in Applied Mechanics and Engineering, 315 (2017) 247-272.

[49] D.P. Kingma, J. Ba, Adam: A method for stochastic optimization, arXiv preprint arXiv:1412.6980, (2014).

[50] S. Ruder, An overview of gradient descent optimization algorithms, arXiv preprint arXiv:1609.04747, (2016).

[51] C. Goldenberg, I. Goldhirsch, Force chains, microelasticity, and macroelasticity, Physical review letters, 89 (2002) 084302.

[52] J.F. Peters, M. Muthuswamy, J. Wibowo, A. Tordesillas, Characterization of force chains in granular material, Phys Rev E Stat Nonlin Soft Matter Phys, 72 (2005) 041307.

[53] Q. Sun, F. Jin, J. Liu, G. Zhang, Understanding force chains in dense granular materials, International Journal of Modern Physics B, 24 (2010) 5743-5759.

[54] T. Qu, Y. Feng, Y. Wang, M. Wang, Discrete element modelling of flexible membrane boundaries for triaxial tests, Computers and Geotechnics, 115 (2019) 103154.

[55] S. Wang, T. Qu, Y. Fang, J. Fu, J. Yang, Stress Responses Associated with Earth Pressure Balance Shield Tunneling in Dry Granular Ground Using the Discrete-Element Method, International Journal of Geomechanics, 19 (2019) 04019060.

[56] D.M. Mueth, H.M. Jaeger, S.R. Nagel, Force distribution in a granular medium, Physical Review E, 57 (1998) 3164.

[57] S. Jiang, Y. Ye, X. Li, S. Liu, J. Liu, D. Yang, Y. Tan, DEM modeling of crack coalescence between two parallel flaws in $\mathrm{SiC}$ ceramics, Ceramics International, 45 (2019) 14997-15014.

[58] X. Li, S. Jiang, Y. Ye, S. Liu, Z. Xu, Y. Tan, D. Yang, Influence of random pore defects on failure mode and mechanical properties of $\mathrm{SiC}$ ceramics under uniaxial compression using discrete element method, Ceramics International, 44 (2018) 22271-22282.

[59] M. Wang, P. Cao, Calibrating the micromechanical parameters of the PFC2D (3D) models using the improved simulated annealing algorithm, Math Probl Eng, 2017 (2017).

[60] Z. Han, D. Weatherley, R. Puscasu, A relationship between tensile strength and loading stress governing the onset of mode I crack propagation obtained via numerical investigations using a bonded particle model, International Journal for Numerical and Analytical Methods in Geomechanics, 41 (2017) 1979-1991.

[61] R. Chen, J. Li, Y. Qian, R. Peng, S. Jiang, C. Hu, Z. Zhao, An Effective Inverse Procedure for Identifying DEMParameters of Rock-Like Materials, Math Probl Eng, 2019 (2019).

[62] S. Zhai, J. Zhan, Y. Ba, J. Chen, Y. Li, Z. Li, PFC model parameter calibration using uniform experimental design and a deep learning network, IOP Conference Series: Earth and Environmental Science, IOP Publishing, 2019, pp. 032062.

[63] L. Scholtès, F.-V. Donzé, A DEM model for soft and hard rocks: role of grain interlocking on strength, Journal of the Mechanics and Physics of Solids, 61 (2013) 352-369. 
[64] A. Fakhimi, Application of slightly overlapped circular particles assembly in numerical simulation of rocks with high friction angles, Engineering Geology, 74 (2004) 129-138.

[65] N.a. Cho, C. Martin, D. Sego, A clumped particle model for rock, International Journal of Rock Mechanics and Mining Sciences, 44 (2007) 997-1010.

[66] M. Xia, C.-b. Zhao, Simulation of rock deformation and mechanical characteristics using clump parallel-bond models, Journal of Central South University, 21 (2014) 2885-2893.

[67] G. Gao, M.A. Meguid, Modeling the impact of a falling rock cluster on rigid structures, International Journal of Geomechanics, 18 (2017) 04017141.

[68] G. Liu, M. Cai, M. Huang, Mechanical properties of brittle rock governed by microgeometric heterogeneity, Computers and Geotechnics, 104 (2018) 358-372.

[69] S. Wu, X. Xu, A study of three intrinsic problems of the classic discrete element method using flat-joint model, Rock Mechanics and Rock Engineering, 49 (2016) 1813-1830.

[70] T. Kazerani, J. Zhao, A microstructure-based model to characterize micromechanical parameters controlling compressive and tensile failure in crystallized rock, Rock mechanics and rock engineering, 47 (2014) 435-452. 


\section{Figures}

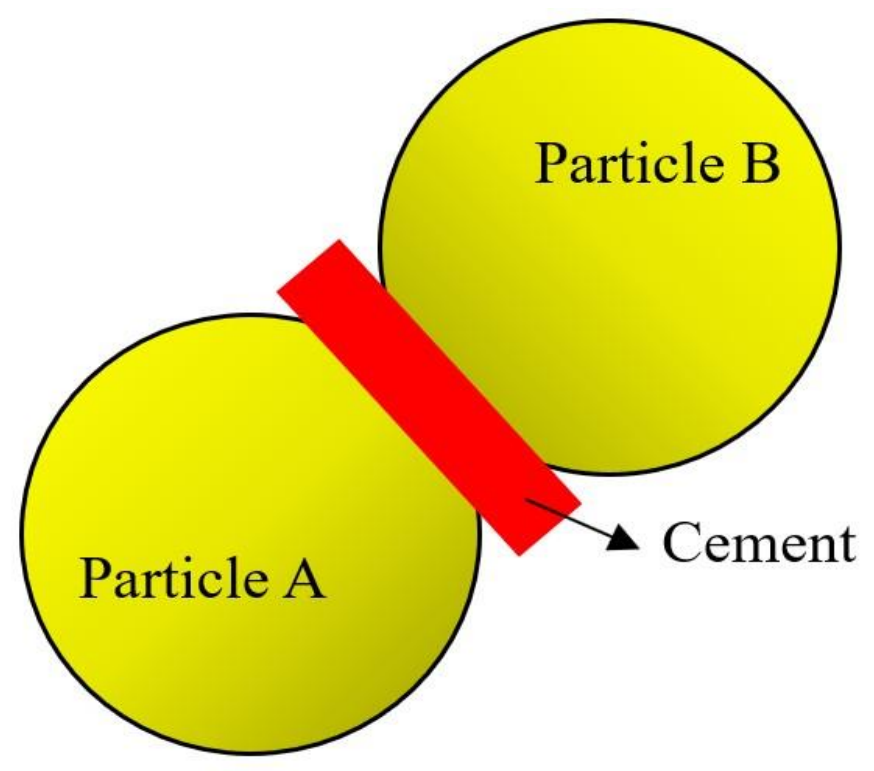

Fig.1 Two particles with the cement between them 


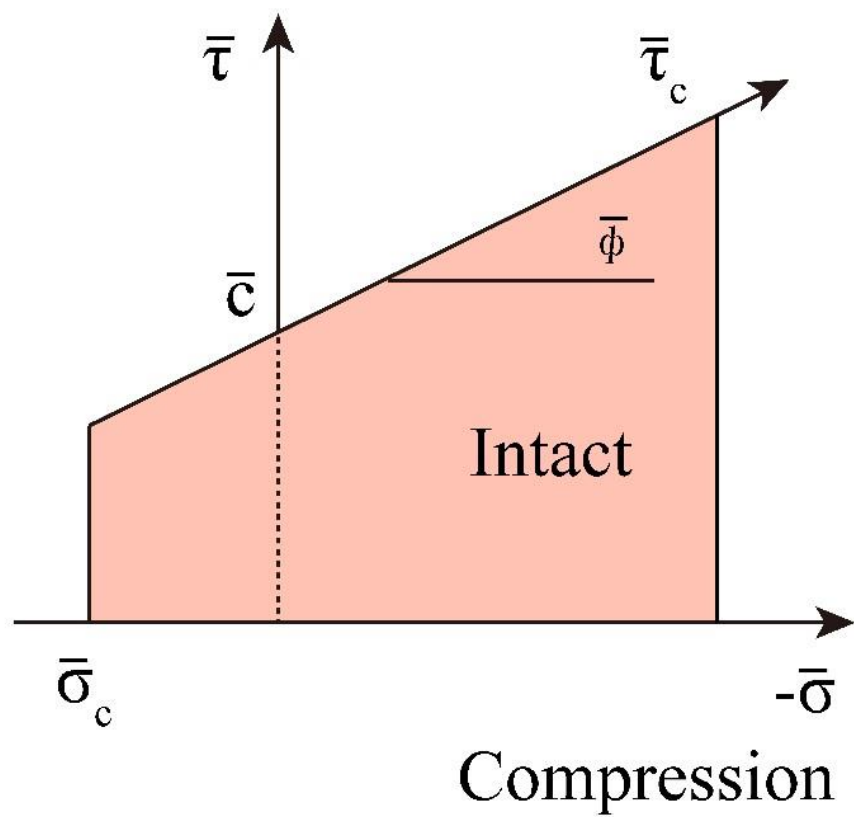

Fig. 2 Failure envelope for the parallel bond 


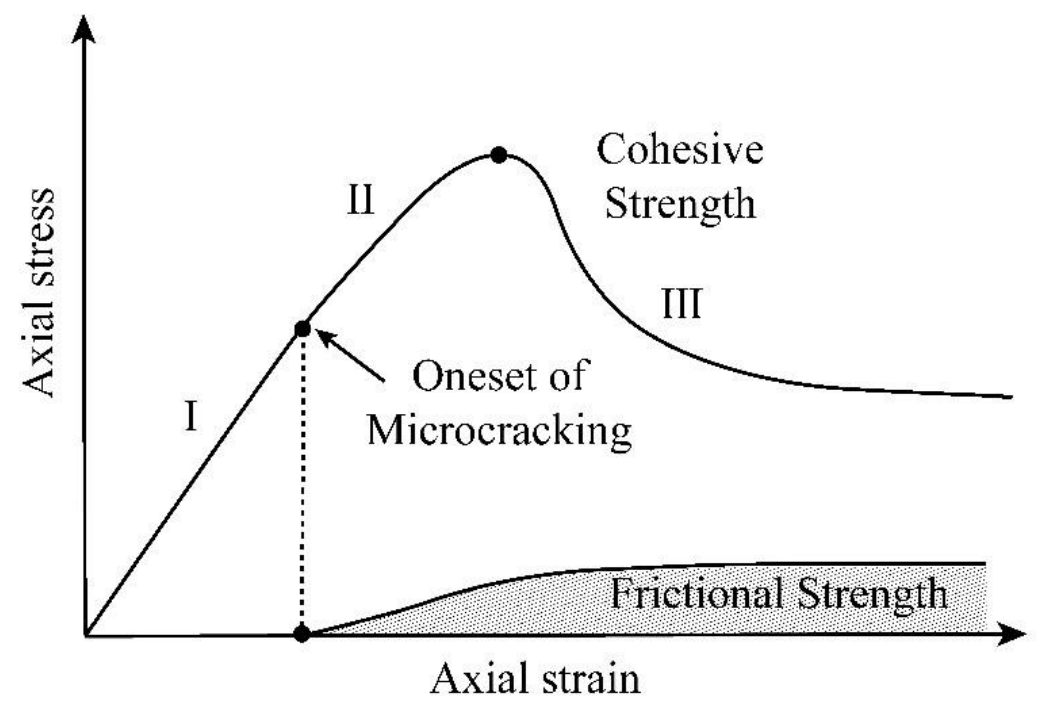

Fig.3 Failure process of a bonded particle model subjected to axial compression (adapted from [39]) 


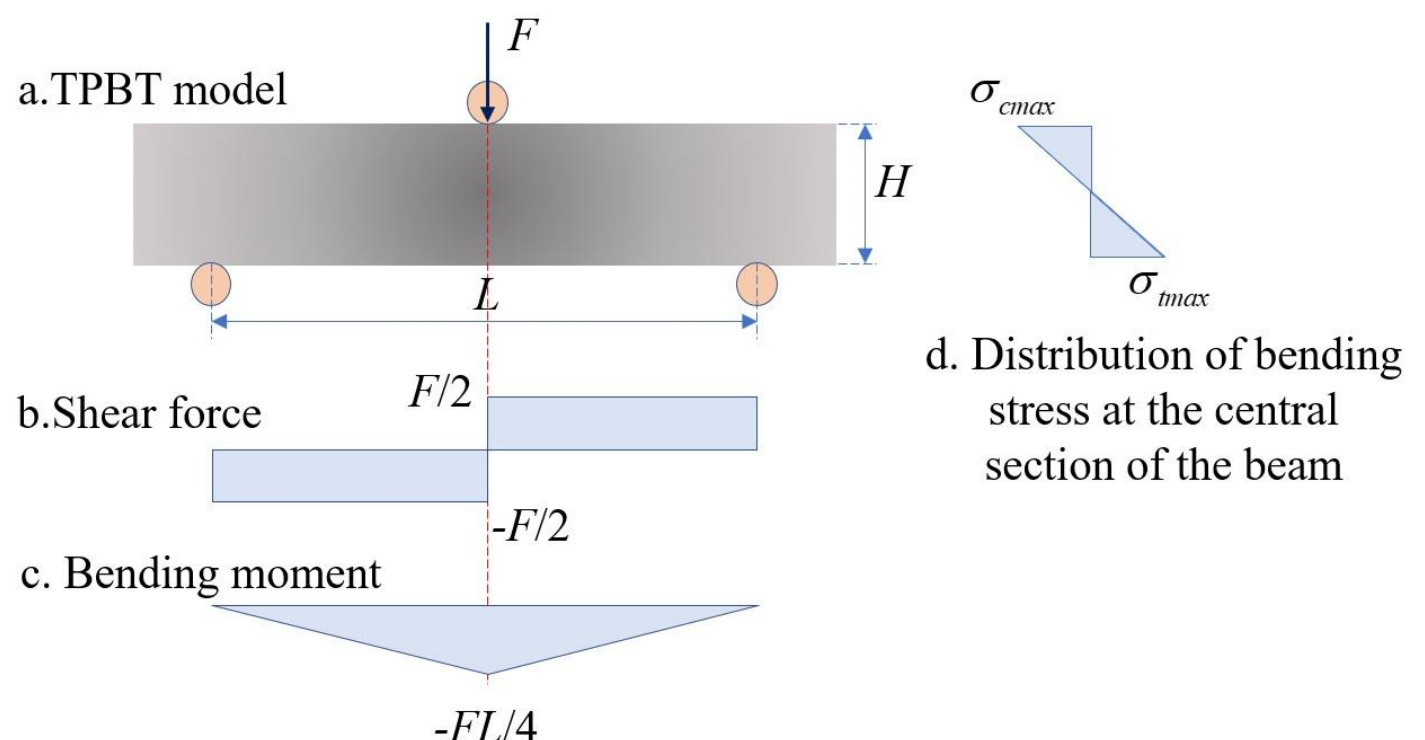

Fig. 4 A beam subjected to three-point bending testing ( $F$ is a load at the central point, $L$ is the support span, $H$ is the height of the specimen, $\sigma_{\text {cmax }}$ and $\sigma_{\text {tmax }}$ denote the maximum compressive stress and tensile stress, respectively) 


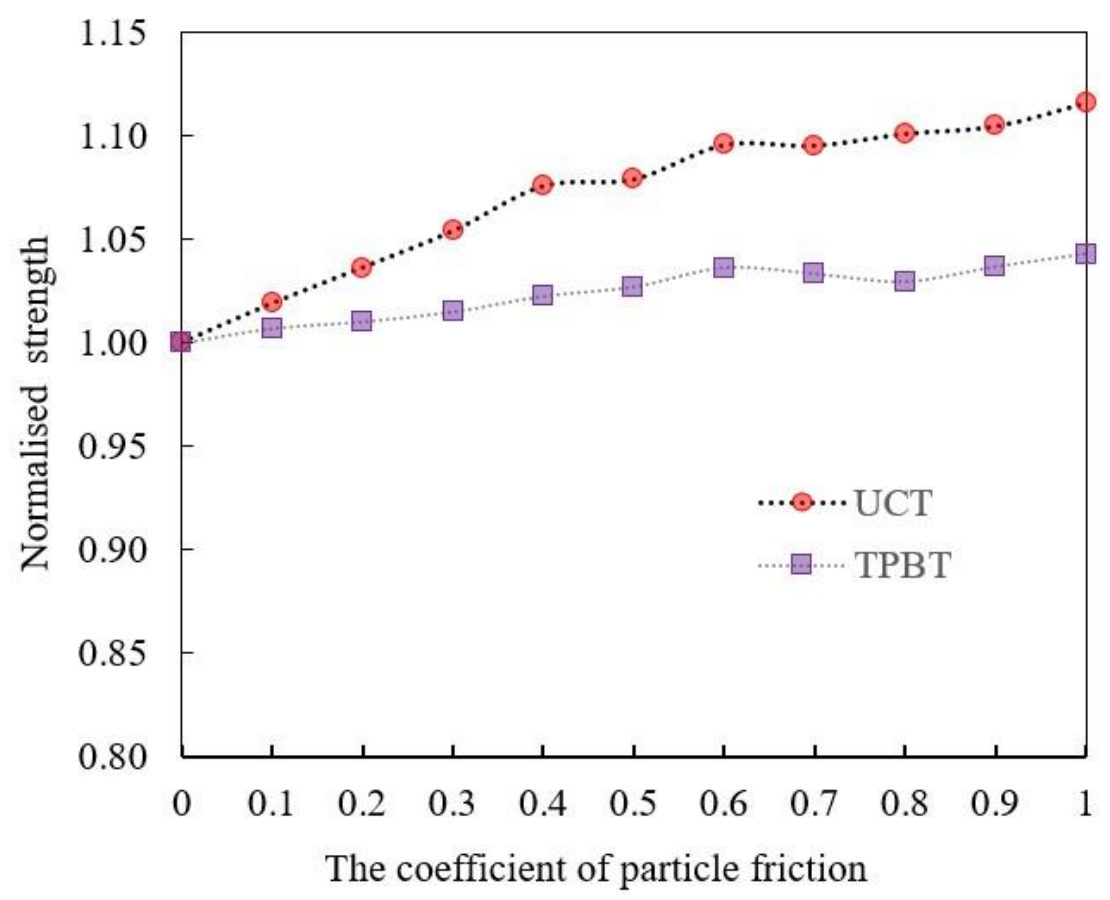

Fig. 5 The influences of particle friction on macroscopic compressive strength and flexural strength 
Experimental flexural strength and compressive strength

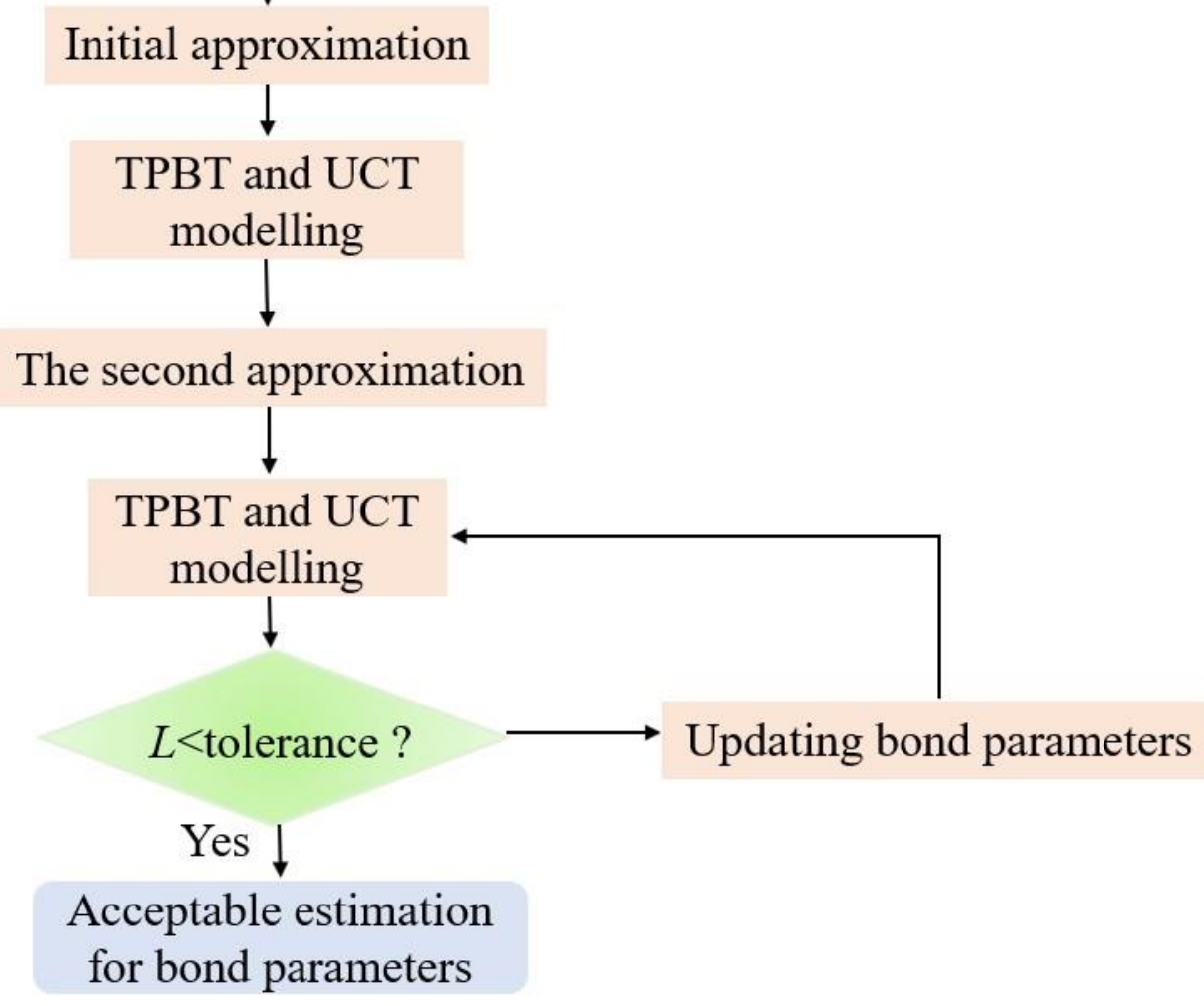

Acceptable estimation for bond parameters 
Fig. 6 Flow chart of the calibration procedure for bond parameters

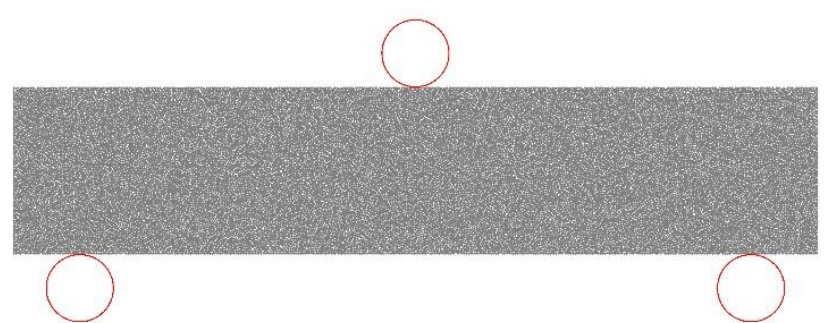

(a) TPBT

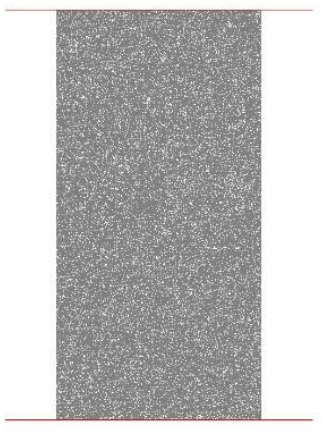

(a) UCT

Fig .7 The numerical models used for bond parameter calibration 


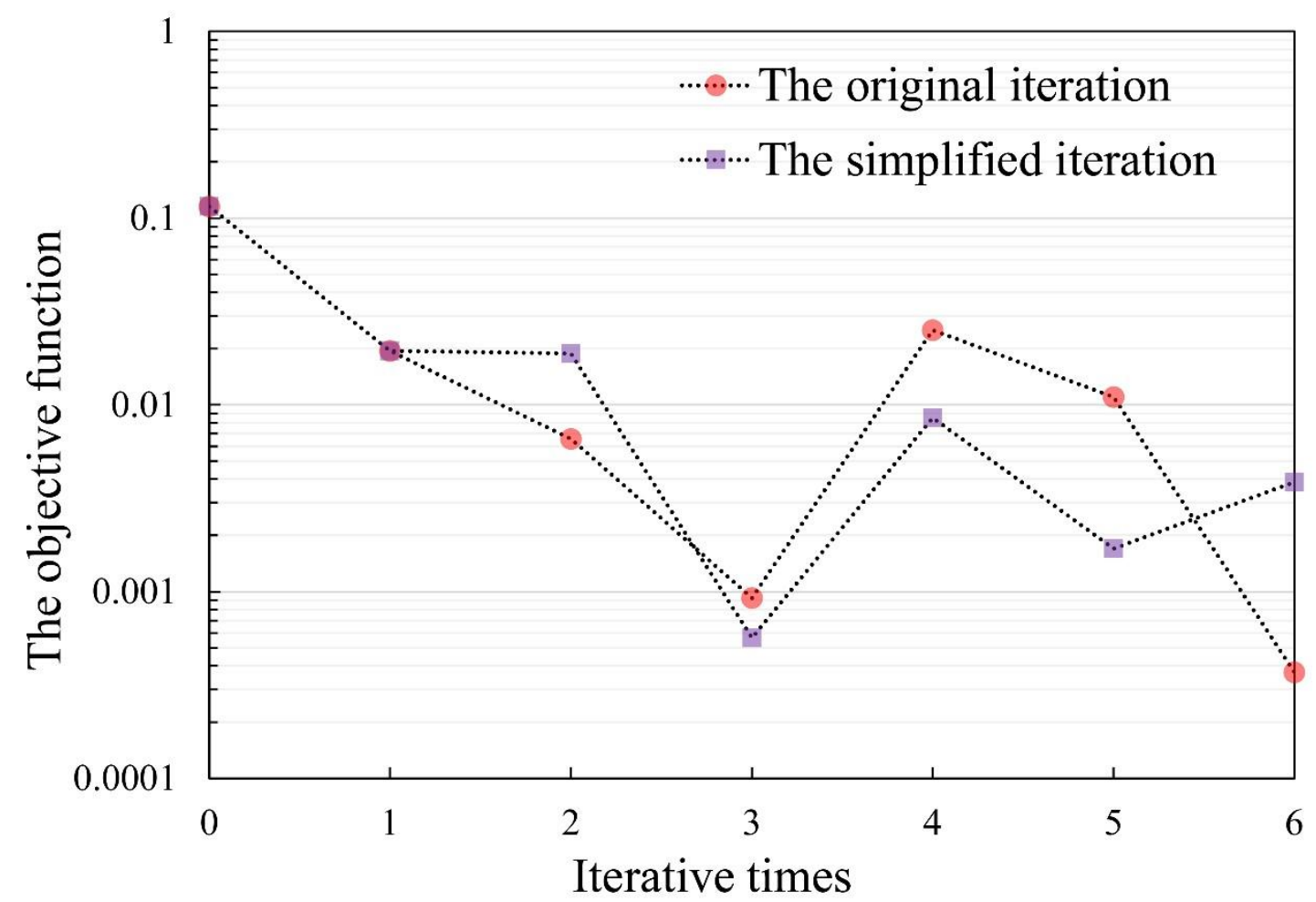

Fig. 8 Evolution of the objective function during iterations 


\section{Tables}

Table 1 Particle-scale parameters and their dimensions (note: $L, F$, and $T$ represent the primary dimensions of length, force, and time, respectively)

\begin{tabular}{|c|c|c|c|c|}
\hline Group & & Parameter & Symbol & Dimension \\
\hline \multirow{4}{*}{$\begin{array}{c}\text { Geometrical } \\
\text { and } \\
\text { physical } \\
\text { parameters }\end{array}$} & \multicolumn{2}{|c|}{ Average particle radius } & $r$ & \multirow[t]{2}{*}{$L$} \\
\hline & \multicolumn{2}{|r|}{ Porosity } & $\phi$ & \\
\hline & \multicolumn{2}{|r|}{ Density } & $\rho$ & $F L-4 T_{2}$ \\
\hline & \multicolumn{2}{|c|}{ Geometry characteristics of the specimen } & $l$ & \\
\hline \multirow{9}{*}{$\begin{array}{c}\text { Constitutive } \\
\text { parameters }\end{array}$} & \multirow{3}{*}{$\begin{array}{c}\text { Grain } \\
\text { parameters }\end{array}$} & Young's modulus of particles & $E_{c}$ & \multirow[t]{3}{*}{$F L-1$} \\
\hline & & $\begin{array}{l}\text { The ratio of shear to normal } \\
\text { stiffness of the grains }\end{array}$ & $k_{s} / k_{n}$ & \\
\hline & & Friction coefficient & $\mu$ & \\
\hline & \multirow{6}{*}{$\begin{array}{c}\text { Cement } \\
\text { parameters }\end{array}$} & Young's modulus of cements & $\overline{E_{c}}$ & $F L^{-2}$ \\
\hline & & $\begin{array}{l}\text { The ratio of shear to normal } \\
\text { stiffness of the cements }\end{array}$ & $k k_{s} / n$ & \\
\hline & & Tensile strength & $\sigma_{\mathrm{c}}$ & $F L^{-2}$ \\
\hline & & Shear strength & $\tau_{\mathrm{c}}$ & $F L^{-2}$ \\
\hline & & Frictional angle & $\phi$ & \\
\hline & & radius multiplier & $\bar{\lambda}$ & \\
\hline
\end{tabular}


Initial or boundary

Table 2 The values of ${ }_{c} /_{c}{ }_{c}$ in several brittle materials

\begin{tabular}{ccc}
\hline Specimen type & 2D model & 3D model \\
\hline Lac du Bonnet granite & $0.764[1]$ & $0.505[11]$, and $0.5[1]$ \\
Beishan (BS) granite & $0.627[42]$, and $0.628[43]$ & $1.0[41]$ \\
red sandstone & $0.551[44]$ & $0.313[45]$ \\
concrete & & $0.5[4]$ \\
sea ice & $0.125[3]$ & \\
SiC ceremics &
\end{tabular}


Table 3 Microscopic parameters used in simulations

\begin{tabular}{|c|c|c|}
\hline Variable & Value & Remarks \\
\hline Particle radius $(\mathrm{m})$ & $4.5 \mathrm{e}-6$ to $6.75 \mathrm{e}-6$ & Uniform distribution \\
\hline Density $\left(\mathrm{kg} / \mathrm{m}^{3}\right)$ & 2600 & \\
\hline Effective modulus (Pa) & $211 \mathrm{e} 9$ & For both particles and bonds \\
\hline $\begin{array}{l}\text { Normal-to-shear stiffness } \\
\text { ratio }\end{array}$ & 1.21 & For both particles and bonds \\
\hline Shear bond strength $(\mathrm{Pa})$ & $7.8 \mathrm{e} 8$ & \\
\hline Tensile bond strength $(\mathrm{Pa})$ & $5 \mathrm{e} 9$ & \\
\hline Initial friction coefficient & 0.7 & $\begin{array}{l}\text { Before loading, the coefficient } \\
\text { of particle friction is changed } \\
\text { from } 0 \text { to } 1.0\end{array}$ \\
\hline Bond gap & $2 \mathrm{e}-7$ & \\
\hline
\end{tabular}


Table 4 The convergence history of the original full version

\begin{tabular}{|c|c|c|c|c|c|c|}
\hline \multirow{4}{*}{$\begin{array}{l}\text { Iterative } \\
\text { numbers }\end{array}$} & \multicolumn{2}{|c|}{ Particle-scale parameters } & \multicolumn{2}{|c|}{ Macroscopic strength } & \multirow[b]{2}{*}{ Flexurat } & \multirow{2}{*}{ Compressive } \\
\hline & & & & & & \\
\hline & tensite- & shear & flexural & compressive & \multirow[b]{2}{*}{ strength error } & \\
\hline & & & & & & strength error \\
\hline & strength (Pa) & strength (Pa) & strength (Pa) & strength (Pa) & & \\
\hline 0 & 780000000 & 2000000000 & 483187287 & 1412539673 & $-38.1 \%$ & $-29.4 \%$ \\
\hline 1 & 1259139087 & 2831778870 & 630918442 & 1901278790 & $-19.1 \%$ & $-4.9 \%$ \\
\hline 2 & 1259291059 & 2831389198 & 808304937 & 1782465166 & $3.6 \%-10.9 \%$ & \\
\hline 3 & 1259329214 & 2831291492 & 763528681 & 1925063036 & $-2.1 \%-3.7 \%$ & \\
\hline 4 & $\begin{array}{l}1259332906 \\
8956994591\end{array}$ & $\begin{array}{l}831286456605 \\
9047486014.8 \%\end{array}$ & $\begin{array}{l}7145520233 \\
-0.5 \%\end{array}$ & $510-22.4 \%$ & $2 \% 512592479$ & 132831572616 \\
\hline 6 & 1259193451 & 2831687578 & 758825095 & 1996876485 & $-2.7 \%$ & $-0.2 \%$ \\
\hline
\end{tabular}


Table 5 The convergence history of the simplified version

\begin{tabular}{|c|c|c|c|c|c|c|}
\hline \multirow{4}{*}{$\begin{array}{l}\text { fterative } \\
\text { numbers }\end{array}$} & \multicolumn{2}{|c|}{ Particle-scale parameters } & \multicolumn{2}{|c|}{ Macroscopic strength } & \multirow{2}{*}{\multicolumn{2}{|c|}{ fommescive- }} \\
\hline & \multirow{3}{*}{ tensite- } & & & & & \\
\hline & & shear & flexural & compressive- & \multirow[b]{2}{*}{ strength error } & \\
\hline & & & & & & strength error \\
\hline & strength (Pa) & strength (Pa) & strength (Pa) & strength (Pa) & & \\
\hline 0 & 780000000 & 2000000000 & 483187287 & 1412539673 & $-38.1 \%-29.4 \%$ & \\
\hline 1 & 1259139087 & 2831778870 & 630918442 & 1901278790 & $-19.1 \%-4.9 \%$ & \\
\hline 2 & \multicolumn{6}{|c|}{ 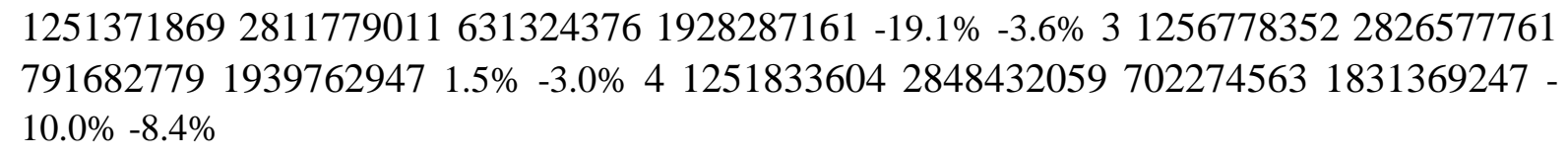 } \\
\hline 5 & 1255654758 & 2861930486 & 820511252 & 2052768684 & $5.2 \% \quad 2.6 \%$ & \\
\hline 6 & 1258923303 & 2873820570 & 772275118 & 1825236495 & $-1.0 \% \quad-8.7 \%$ & \\
\hline
\end{tabular}


Table 6 Comparison of efficiency and accuracy for different calibration methods

\begin{tabular}{|c|c|c|c|c|}
\hline \multirow{3}{*}{ Method } & Calibration & \multirow{3}{*}{ Calibration error } & \multirow{3}{*}{ Efficiency } & \multirow{3}{*}{$\begin{array}{l}\text { Publish } \\
\text { year }\end{array}$} \\
\hline & & & & \\
\hline & experiments & & & \\
\hline $\begin{array}{l}\text { A hybrid DEM codes and SNOBFIT } \\
\text { optimisation method [11] }\end{array}$ & Triaxial tests & $5 \%$ & $\begin{array}{l}95 \text { iterations, } \\
570 \text { models }\end{array}$ & 2010 \\
\hline $\begin{array}{c}\text { Improved Simulated Annealing } \\
\text { Algorithm [59] }\end{array}$ & UCT & $9.4 \%$ & 2739 iterations & 2017 \\
\hline Surface fitting method [60] & Brazilian tests & $10 \%$ & 80 models & 2017 \\
\hline $\begin{array}{l}\text { Ant-colony optimization algorithm } \\
{[61]}\end{array}$ & TPBT and UCT & $5 \%$ (UCT), $10 \%$ (TPBT) & 200 iterations & 2019 \\
\hline Surface fitting method [14] & $\begin{array}{l}\text { Uniaxial tensile } \\
\text { test }\end{array}$ & $\begin{array}{c}1 \%-7 \% \text { (Brizilian test), } 2 \% \\
\text { (UCT) }\end{array}$ & 6400 models & 2019 \\
\hline Deeping learning network [62] & $\begin{array}{l}\text { UCT, Direct } \\
\text { tensile test, } \\
\text { Triaxial test }\end{array}$ & $\begin{array}{c}3.493 \%-12.031 \%(\mathrm{UCT}) \\
1.527 \% \text { to } 27.985 \% \\
\text { (Triaxial test ) }\end{array}$ & 288 models & 2019 \\
\hline $\begin{array}{l}\text { Physics-informed adaptive moment } \\
\text { optimization (the original iterative } \\
\text { scheme) }\end{array}$ & UCT and TPBT & $\begin{array}{l}2.7 \%(\mathrm{TPBT}) \text { and } 1.6 \% \\
\text { (UCT) }\end{array}$ & $\begin{array}{l}6 \text { iterations, } 19 \\
\text { models }\end{array}$ & \\
\hline $\begin{array}{l}\text { Physics-informed adaptive moment } \\
\text { optimization (the simplified iterative } \\
\text { scheme) }\end{array}$ & UCT and TPBT & $\begin{array}{c}1.5 \%(\mathrm{TPBT}) \text { and } 3.0 \% \\
\text { (UCT) }\end{array}$ & $\begin{array}{l}3 \text { iterations, } 4 \\
\text { models }\end{array}$ & \\
\hline
\end{tabular}

